\title{
Ion-hose instability in a long-pulse linear induction accelerator
}

\author{
Thomas C. Genoni* and Thomas P. Hughes ${ }^{\dagger}$ \\ Mission Research Corporation, 5001 Indian School Road NE, Albuquerque, New Mexico 87110
}

(Received 25 November 2002; published 19 March 2003)

\begin{abstract}
The ion-hose instability is a transverse electrostatic instability which occurs on electron beams in the presence of a low-density ion channel. It is a phenomenon quite similar to the interaction between electron clouds and proton or positron beams in high-energy accelerators and storage rings. In the DARHT-2 accelerator, the 2-kA, 2- $\mu$ s beam pulse produces an ion channel through impact ionization of the residual background gas $\left(10^{-7}-10^{-6}\right.$ torr). A calculation of the linear growth by Briggs indicates that the instability could be strong enough to affect the radiographic application of DARHT, which requires that transverse oscillations be small compared to the beam radius. We present semianalytical theory and 3D particle-in-cell simulations (using the LSP code) of the linear and nonlinear growth of the instability, including the effects of the temporal change in the ion density and spatially decreasing beam radius. We find that the number of $e$-foldings experienced by a given beam slice is given approximately by an analytic expression using the local channel density at the beam slice. Hence, in the linear regime, the number of $e$-foldings increases linearly from head to tail of the beam pulse since it is proportional to the ion density. We also find that growth is strongly suppressed by nonlinear effects at relatively small oscillation amplitudes of the electron beam. This is because the ion oscillation amplitude is several times larger than that of the beam, allowing nonlinear effects to come into play. An analogous effect has recently been noted in electron-proton instabilities in high-energy accelerators and storage rings. For DARHT-2 parameters, we find that a pressure of $\leq 1.5 \times 10^{-7}$ torr is needed to keep the transverse beam oscillation amplitude less than about $20 \%$ of the rms beam radius.
\end{abstract}

DOI: 10.1103/PhysRevSTAB.6.030401

PACS numbers: 29.27.-a, 41.75.-i, 52.35.-g, 52.59.-f

\section{INTRODUCTION}

The DARHT-2 accelerator [1] (Fig. 1) is designed to produce a $2-\mathrm{kA}, 20-\mathrm{MV}, 2-\mu$ s flattop electron beam pulse guided by a focusing solenoidal magnetic field. It is expected that electron impact ionization of the residual background gas in the accelerator $\left(10^{-7}-10^{-6}\right.$ torr $)$ will result in an ion population with a line charge density of order $10^{-4}-10^{-3}$ of the beam line charge density. Even at this relatively low ion density, significant linear growth of the transverse ion-hose instability is possible, as pointed out by Briggs [2]. In this article, semianalytical theory and 3D particle-in-cell (PIC) simulation are used to investigate the potential severity of this instability in DARHT-2. In Sec. II, Lee's "spread-mass" model [3], as applied previously to the ion-hose instability [4], is extended to include the effect of a focusing magnetic field. The growth of the instability for typical DARHT-2 parameters is calculated from the linear theory. We also make use of a nonlinear ansatz to simplify the numerical calculations. The latter predict that nonlinear effects can decrease the growth rate significantly at small beam oscillation amplitudes. A recent article by Channell [5] discusses a similar phenomenon for the electron-proton $(e-p)$ instability [6] in a proton storage ring. For the $e-p$ instability he observes that the electron oscillations reach nonlinear amplitudes for relatively small amplitudes of

\footnotetext{
*Electronic address: genoni@mrcabq.com
}

†Electronic address: tph@mrcabq.com
$1098-4402 / 03 / 6(3) / 030401(10) \$ 20.00$ the proton beam. In the ion-hose instability the roles of the electrons and ions are reversed. We find that the transverse ion oscillations become nonlinear when the electron beam oscillation amplitude is still relatively small. This causes a large nonlinear reduction in the growth rate of the beam oscillations.

In Sec. III, we describe a PIC model which allows us to carry out self-consistent simulations of the beamchannel interaction over the long spatial (50-m) and temporal $(2-\mu \mathrm{s})$ dimensions required. In Sec. IV, we compare the spread-mass and PIC models for the case of a constant-energy beam in a constant axial magnetic field. In Sec. V, PIC calculations are applied to a configuration similar to the DARHT-2 accelerator, with acceleration from 4 to $20 \mathrm{MV}$, a spatially varying magnetic field, and background gas composition determined experimentally. Conclusions are given in Sec. VI.

\section{SPREAD-MASS MODEL OF ION-HOSE INSTABILITY}

Consider a system composed of an azimuthally symmetric relativistic $(v \simeq c)$ electron beam of current $I_{b}$ and radius $R$, and a coaxial ion channel of equal radius produced by impact ionization of the background gas. The transverse displacements of the beam and channel with respect to the original equilibrium position are denoted by $b$ and $d$, respectively. We start from the "rigid beam" equations $[4,7]$ for ion-hose oscillations 


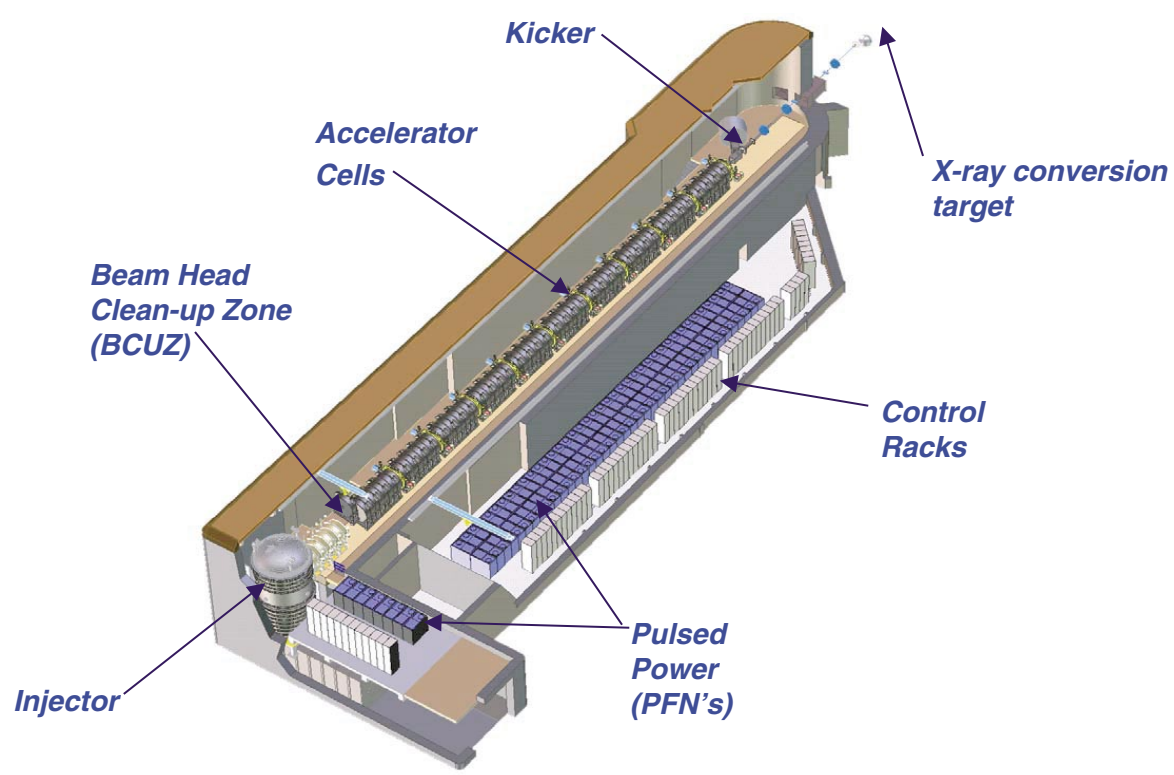

FIG. 1. (Color) Schematic of DARHT-2 accelerator.

$$
\begin{aligned}
& \frac{\partial^{2} b}{\partial z^{2}}=-k_{\beta e}^{2}(b-d)+i k_{c e} \frac{\partial b}{\partial z}, \\
& \frac{\partial^{2} d}{\partial \tau^{2}}=-\omega_{\beta i}^{2}(d-b)-i \omega_{c i} \frac{\partial d}{\partial \tau},
\end{aligned}
$$

where $b$ and $d$ are complex phasors defined by $b=b_{x}+$ $i b_{y}$ and $d=d_{x}+i d_{y}$. In Eqs. (1) and (2), $z$ measures distance along the accelerator and $\tau=t-z / c$ labels a particular beam segment by its time of injection at $z=0$. With the assumption that both the beam and the channel are Gaussian in profile, the electron betatron wave number and ion oscillation frequency are given, respectively, by

$$
\begin{gathered}
k_{\beta e}^{2}=\frac{f \nu}{\gamma R^{2}}, \\
\omega_{\beta i}^{2}=\frac{m_{e} \nu c^{2}}{m_{i} R^{2}},
\end{gathered}
$$

where $m_{e}$ and $m_{i}$ are the electron and ion masses, $\gamma$ is the electron relativistic factor, $\nu=e I_{b} / m c^{3}$, and $f$ is the fractional neutralization. (In the dispersion analysis which follows we make the simplifying assumption of constant $f$. For the case of beam-generated ionization, one would expect $f$ at any position $z$ to ramp up roughly linearly with $\tau$. This case is considered in the numerical calculations later in this section and in the simulations of Sec. III.) The electron cyclotron wave number $k_{c e}$ and the ion cyclotron frequency $\omega_{c i}$ are defined by

$$
k_{c e}=\frac{e B_{z}}{\gamma m_{e} c^{2}},
$$

$$
\omega_{c i}=\frac{e B_{z}}{m_{i} c},
$$

with $B_{z}$ the solenoidal focusing magnetic field.

Following Buchanan [4], we incorporate frequency spreading by using the spread-mass model of Lee [3]. Each longitudinal segment of the beam and channel is divided into rigid disks having varying masses and labeled by the continuous variables $\eta$ and $\xi(0 \leq \eta, \xi \leq$ 1 ). The displacement of any beam (or channel) segment is the weighted mean of all the disks; i.e.,

$$
\begin{aligned}
& \bar{B}=\int_{0}^{1} g(\eta) B_{\eta} d \eta, \\
& \bar{D}=\int_{0}^{1} g(\xi) D_{\xi} d \xi,
\end{aligned}
$$

where $g(x)=12 x^{2}(1-x)$ is the normalized weighting function appropriate to the Gaussian profile [3]. Making use of Eqs. (7) and (8) and defining the scaled quantities $Z=k_{\beta e} z, T=\omega_{\beta i} \tau, K_{c e}=k_{c e} / k_{\beta e}$, and $\Omega_{c i}=\omega_{c i} / \omega_{\beta i}$, we arrive at the spread-mass model equations for ionhose oscillations in the presence of a focusing magnetic field:

$$
\begin{aligned}
& \frac{\partial^{2} B_{\eta}}{\partial Z^{2}}=-2 \eta\left(B_{\eta}-\bar{D}\right)+i K_{c e} \frac{\partial B_{\eta}}{\partial Z} \quad(0 \leq \eta \leq 1) \\
& \frac{\partial^{2} D_{\xi}}{\partial T^{2}}=-2 \xi\left(D_{\xi}-\bar{B}\right)-i \Omega_{c i} \frac{\partial D_{\xi}}{\partial T} \quad(0 \leq \xi \leq 1) .
\end{aligned}
$$

For a Gaussian beam and channel, the maximum (on-axis) values of the squares of the betatron wave 
number and ion frequency are twice the rigid beam value given in Eqs. (3) and (4), giving rise to the factors of 2 in Eqs. (9) and (10).

We next assume harmonic perturbations for $B_{\eta}$ and $D_{\xi}$ of the form

$$
B_{\eta}, D_{\xi} \sim e^{i(\omega t-\kappa z)}=e^{i(\omega \tau+k z)}=e^{i(\Omega T+K Z)},
$$

where $\kappa$ is the laboratory wave number, $k=\omega / c-\kappa$ is the "Doppler-shifted" wave number, and $K=k / k_{\beta e}$ and $\Omega=\omega / \omega_{\beta i}$ are the scaled wave number and frequency. Substitution into Eqs. (9) and (10) and use of the definitions in Eqs. (7) and (8) result in the set of equations

$$
\begin{aligned}
& \frac{\bar{B}}{\bar{D}}=\int_{0}^{1} \frac{2 \eta g(\eta) d \eta}{[2 \eta-U(K)]}, \\
& \overline{\bar{D}}=\int_{0}^{1} \frac{2 \xi g(\xi) d \xi}{[2 \xi-W(\Omega)]},
\end{aligned}
$$

and finally the dispersion relation

$$
\int_{0}^{1} \frac{2 \eta g(\eta) d \eta}{[2 \eta-U(K)]} \int_{0}^{1} \frac{2 \xi g(\xi) d \xi}{[2 \xi-W(\Omega)]}=1
$$

where $U(K)=K^{2}-K K_{c e}$ and $W(\Omega)=\Omega^{2}+\Omega \Omega_{c i}$. The integrations in Eq. (14) may be carried out to give the dispersion relation in the form

$$
f\left[\frac{U(K)}{2}\right] f\left[\frac{W(\Omega)}{2}\right]=1,
$$

where

$$
\begin{aligned}
f(x)= & 1+2 x+6 x^{2}-12 x^{3}+12\left(x^{3}-x^{4}\right) \\
& \cdot \log [(x-1) / x] .
\end{aligned}
$$

At a fixed downstream position $z$ (e.g., the end of the accelerator), the growth of the instability is bounded according to

$$
|\bar{B}| \leq C_{0} e^{K_{0} Z}=C_{0} e^{\Gamma},
$$

where $C_{0}$ is a constant and $K_{0}$ is the maximum of the imaginary part of $(-K)$ for all real $\Omega$. We see from inspection of Eq. (14) that in the large $B_{z}$ limit $\left(K_{c e} \gg K\right)$, this occurs approximately when $\operatorname{Im}[U(K)]$ is maximized. Neglecting the ion cyclotron frequency $\Omega_{c i}$ (it is negligible for the range of parameters considered here), we find from the numerical solution of Eq. (15) that

$$
K_{c e} \max [\operatorname{Im}(-K)]_{\mathrm{real} \Omega} \simeq \max [\operatorname{Im}(U)]_{\mathrm{real} \Omega} \simeq 4.75
$$

at $\Omega_{0}=1.22$. The instability is convective, and the point at which linear saturation is reached (for a resonant sinusoidal excitation) moves back from the head of the beam and is given approximately by

$$
T_{\text {sat }}=\left[-\left.\frac{\partial K_{r}}{\partial \Omega}\right|_{\Omega=\Omega_{0}}\right]^{-1} Z \simeq \frac{23.7}{K_{c e}} Z .
$$

It follows that the growth factor at the end of the accelerator may be approximated for large $B_{z}$ as

$$
\Gamma \simeq \frac{4.75 Z_{\max }}{K_{c e}}=\frac{4.75 f \nu m_{e} c^{2} \ell}{e B_{z} R^{2}} .
$$

Although the exact numerical solution of Eq. (15) is straightforward, Eq. (19) provides a reasonable approximation for DARHT-2 and it explicitly displays how the growth scales with parameters of interest. We note that when the beam radius is in the emittance-dominated regime [see Eq. (32)], the amplification factor becomes

$$
\Gamma \simeq \frac{4.7 f \nu l}{\varepsilon},
$$

where $\varepsilon$ is the normalized "edge" emittance. Since the accelerator requirements on DARHTare given in terms of beam current and emittance, we see from Eq. (20) that modifying the tune does not change the instability growth in this region.

We next consider the implications for a typical set of DARHT-2 parameters: $I_{b}=2 \mathrm{kA}, \gamma_{A V}=25, R=$ $0.5 \mathrm{~cm}, B_{z}=830 \mathrm{G}, \ell=50 \mathrm{~m}$, and $p=2 \times 10^{-7}$ torr. Residual-gas analysis of an evacuated DARHT-2 cell [8] showed $\mathrm{H}_{2} \mathrm{O}$ to be the dominant species. Making use of the cross section for electron impact ionization of $\mathrm{H}_{2} \mathrm{O}$ (it is $\approx 0.9 \times 10^{-18} \mathrm{~cm}^{2}$ in the range 4 to $20 \mathrm{MeV}$ ), we obtain the following expression for the fractional neutralization $f$ :

$$
f \simeq 0.9 \times 10^{9} \cdot p(\text { torr }) \cdot \tau(\mathrm{s}) .
$$

We first make the "worst case" assumption that $f$ is constant at its maximum value $3.6 \times 10^{-4}$ corresponding to $\tau=2 \mu \mathrm{s}$ at the tail of the beam. Substitution into Eq. (19) gives $\Gamma=8.26$, or slightly more than eight $e$-foldings - a somewhat pessimistic first estimate. Direct numerical solution of Eq. (15) gives $\Gamma=7.69$ (at $\Omega_{0}=1.20$ ) and we see that the large $B_{z}$ limit of Eq. (19) is a modest overestimate for this parameter set. The time to saturation is approximately $360 \mathrm{~ns}$ from Eq. (18).

Equations (9) and (10) were solved numerically for the above set of parameters and with the mass spread over 50 disks. The beam was given a transverse sinusoidal excitation at $z=0$ at the normalized resonant frequency $(21.6 \mathrm{MHz})$. The result is shown as curve $(a)$ in Fig. 2 where we see that the amplitude of the beam displacement at the end of the accelerator grows over the first few hundred ns of the beam to a steady-state value corresponding to slightly more than seven $e$-foldings, consistent with the time to saturation and the amplification upper bound from the dispersion analysis. The more realistic assumption of the linearly ramped channel density requires insertion of the factor $\left(T / T_{\max }\right)$ in the first term on the right-hand side of Eq. (9). ( $T_{\max }=\omega_{\beta i} \tau_{\max }$ where $\tau_{\max }=2 \mu \mathrm{s}$ at the tail of the beam.) This additional factor severely complicates the dispersion analysis but is a trivial modification to the numerical integration of the coupled equations and results in curve $(b)$ of Fig. 2 . We see 


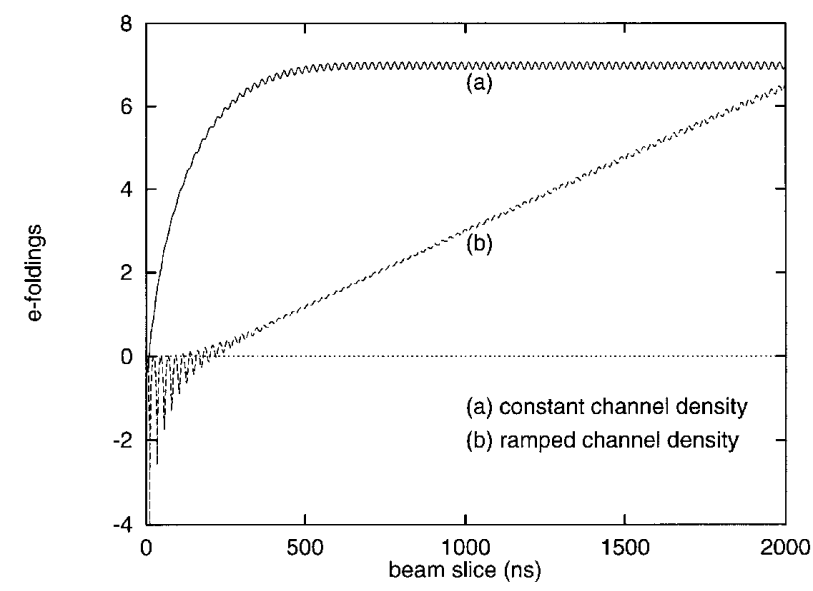

FIG. 2. Amplitude of beam displacement at the end of the accelerator $(\ell=50 \mathrm{~m})$ for $(a)$ constant channel density and (b) linear ramp channel density (from numerical integration of linear model equations).

that for the linearly ramped channel, the amplitude of the beam displacement grows approximately exponentially from beam head to tail to a maximum value only slightly less than the maximum steady-state value for the constant-channel density case. To a good approximation, an expression for the growth in the presence of a temporally increasing density channel is obtained by using the analytic constant-channel expression. The approximate number of $e$-foldings experienced by a beam slice at time $T$ back from the head is obtained by substituting $f=$ $f\left(T_{\max }\right) T / T_{\max }$ into Eq. (19). This is due to the fact that a given slice sees a constant value of $f$ as it moves down the accelerator, and that $f$ is approximately constant in the neighborhood of a given slice. This approximation improves as $T$ increases.

At this point we note that the linear theory provides an estimate for the ratio of the amplitudes of the channel and beam displacements. Substituting $\Omega=1.20$ in Eq. (13) we find that

$$
\frac{|\bar{D}|}{|\bar{B}|} \simeq 4
$$

and this relationship is borne out by the numerical results shown in Fig. 3.

We should expect nonlinear effects to become prominent for beam-channel displacements of the order of the radius $R$, and in light of Eq. (22), for beam displacements as small as perhaps $10 \%-20 \%$ of the radius. This observation prompts us to attempt an extension of our numerical calculations to the nonlinear regime. We note that for our assumed Gaussian profiles, direct calculation [4] of the restoring force $F$ on channel disk $\xi$ due to the beam gives

$$
F \propto \sum_{\eta} g(\eta) \frac{\left[1-e^{-\left|D_{\xi}-B_{\eta}\right|^{2}}\right]}{\left|D_{\xi}-B_{\eta}\right|^{2}}\left(D_{\xi}-B_{\eta}\right),
$$

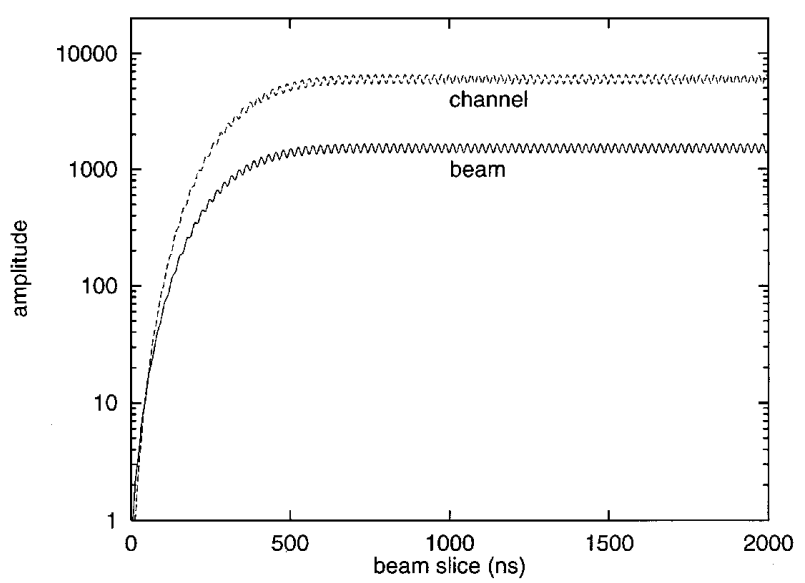

FIG. 3. Amplitudes of channel and beam displacements at the end of the accelerator from numerical integration of linear model equations.

where $D_{\xi}$ and $B_{\eta}$ are scaled to $\sqrt{2} R$. A similar expression obtains for the force on a beam disk $\eta$, giving rise to the nonlinear equations

$$
\begin{aligned}
\frac{\partial^{2} B_{\eta}}{\partial Z^{2}}= & -2 \eta \sum_{\xi} g(\xi) \frac{\left[1-e^{-\left|B_{\eta}-D_{\xi}\right|^{2}}\right]}{\left|B_{\eta}-D_{\xi}\right|^{2}}\left(B_{\eta}-D_{\xi}\right) \\
& +i K_{c e} \frac{\partial B_{\eta}}{\partial Z}, \\
\frac{\partial^{2} D_{\xi}}{\partial T^{2}}= & -2 \xi \sum_{\eta} g(\eta) \frac{\left[1-e^{-\left|D_{\xi}-B_{\eta}\right|^{2}}\right]}{\left|D_{\xi}-B_{\eta}\right|^{2}}\left(D_{\xi}-B_{\eta}\right) \\
& -i \Omega_{c i} \frac{\partial D_{\xi}}{\partial T} .
\end{aligned}
$$

With the simplifying ansatz

$$
F \propto \frac{\left[1-e^{-\left|D_{\xi}-\bar{B}\right|^{2}}\right]}{\left|D_{\xi}-\bar{B}\right|^{2}}\left(D_{\xi}-\bar{B}\right),
$$

our nonlinear version of Eq. (10) becomes

$$
\frac{\partial^{2} D_{\xi}}{\partial T^{2}}=-2 \xi \frac{\left[1-e^{-\left|D_{\xi}-\bar{B}\right|^{2}}\right]}{\left|D_{\xi}-\bar{B}\right|^{2}}\left(D_{\xi}-\bar{B}\right)-i \Omega_{c i} \frac{\partial D_{\xi}}{\partial T} .
$$

Similarly, Eq. (9) becomes

$$
\frac{\partial^{2} B_{\eta}}{\partial Z^{2}}=-2 \eta \frac{\left[1-e^{-\left|B_{\eta}-\bar{D}\right|^{2}}\right]}{\left|B_{\eta}-\bar{D}\right|^{2}}\left(B_{\eta}-\bar{D}\right)+i K_{c e} \frac{\partial B_{\eta}}{\partial Z} .
$$

Numerical integration of Eqs. (27) and (28) for the same parameter set and with the same excitation at $z=0$ produced the results shown in Fig. 4. [A few numerical trials showed that the simplified equations produced results within about $10 \%$ of the full Eqs. (24) and (25).] The model predicts that nonlinear effects cause a large reduction in the linear growth rate. In this case $(p=2 \times$ $10^{-7}$ torr), the instability growth slows dramatically 


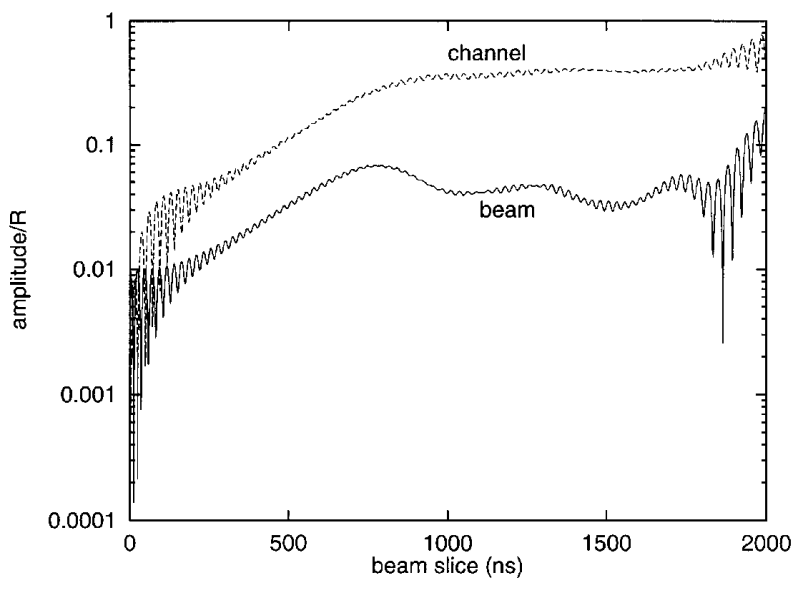

FIG. 4. Amplitude of the beam and channel displacements (in units of the beam radius $R$ ) from numerical integration of the nonlinear model equations.

when the beam displacement is just $10 \%$ of the rms radius $R$. Larger displacements than this initial "saturation" value are seen towards the end of the calculation, near $2 \mu \mathrm{s}$. Calculations at several other pressures further suggest that the initial saturation level is roughly proportional to the pressure, and, hence, to the linear growth rate. These tentative conclusions were investigated more quantitatively in a series of numerical simulations described in the following section.

\section{PIC SIMULATION MODEL OF ION-HOSE INSTABILITY}

The spread-mass model is based on a simplified treatment of beam dynamics. To check this model and include some additional effects, we have used the PIC simulation code LSP [9]. The LSP simulations incorporate the following features, which are absent from the spread-mass model:

(i) Self-consistent treatment of the beam and ion distributions in the self and applied fields. The spread-mass model represents the beam as a collection of rigid disks. Thus, the effect of instabilities on the beam profile and emittance can be modeled with LSP.

(ii) Continuous generation of ions through impact ionization by beam particles on the background gas. Thus, newly created ions have the same centroid position as the beam. The spread-mass model treats the beam ionization by linearly increasing the charge "weight" of a fixed number of rigid ion disks. Thus, newly created ions have the same centroid position as the existing ions.

The simulations retain some assumptions because of the lengthy spatial and temporal scales involved $(50 \mathrm{~m}$ and $2 \mu \mathrm{s}$, respectively):

(iii) Secondary electrons produced in the impact ionization can be neglected.

(iv) Longitudinal forces can be neglected.
The first assumption may not be very good. Because of the strong axial magnetic field, the Larmor radius of the secondary electrons is of the order of a few $\mathrm{cm}$ : larger than the beam radius, but smaller than the pipe radius. The large time steps taken in the simulations do not permit us to temporally resolve these orbits. Previous work [10] has shown that a diffuse electron background tends to mitigate the ion-hose instability by shorting out some of the transverse electric field. On the other hand, longitudinal and transverse beam-electron instabilities may arise [10]. We will not consider these effects further in this article.

The second assumption is justified by the transverse, long-wavelength, low-frequency nature of the ion-hose instability [4]. The transverse electric and magnetic fields are calculated from Poisson-type equations:

$$
\begin{gathered}
\nabla_{\perp}^{2} \phi=4 \pi e\left(n_{b}-n_{i}\right), \\
\nabla_{\perp}^{2} A_{z}=4 \pi n_{b} e v_{z},
\end{gathered}
$$

where $\phi, A_{z}$ are the electrostatic and longitudinal vector potentials, respectively; $n_{b}, n_{i}$ are the beam and ion charge densities, respectively; and $v_{z}$ is the axial beam velocity. These equations are solved using $2 \mathrm{D}$ fast Fourier transforms. Particles are pushed in the transverse direction using the Lorentz equation:

$$
\frac{d \vec{p}_{\perp}}{d t}=q\left(\vec{E}_{\perp}+\frac{v_{z}}{c} \hat{z} \times \vec{B}_{\perp}+\frac{\vec{v}_{\perp}}{c} \times B_{z} \hat{z}\right),
$$

where $v_{z}$ is assumed to be constant. For the ions, the $\vec{v} \times \vec{B}$ force can be neglected to a good approximation.

\section{COMPARISON OF PIC SIMULATIONS WITH SPREAD-MASS RESULTS FOR COASTING BEAM}

We use a series of calculations to compare the PIC and spread-mass models. The beam energy is constant (no acceleration) and a uniform axial magnetic field is used. Parameters are given in Table I. For the LSP simulations, these beam parameters are consistent with a normalized (edge) emittance of $0.1 \mathrm{~cm}$ rad. In the spread-mass model, there is no equilibrium force-balance condition because of the rigid-disk assumption.

TABLE I. Parameters for comparison of PIC and spreadmass models.

\begin{tabular}{lc}
\hline \hline \multicolumn{1}{c}{ Parameters } & Values \\
\hline Beam relativistic factor $\gamma$ & $25(\simeq 12 \mathrm{MeV})$ \\
Beam current & $2 \mathrm{kA}$ \\
Beam pulse length & $2 \mu \mathrm{s}$ \\
Beam rms radius & $0.5 \mathrm{~cm}$ \\
Axial magnetic field & $830 \mathrm{G}$ \\
Transport distance & $50 \mathrm{~m}$ \\
Vacuum pressure range $\left(\mathrm{H}_{2} \mathrm{O}\right)$ & $1-6 \times 10^{-7}$ torr \\
\hline
\end{tabular}


We carry out six simulations, with vacuum pressures from $10^{-7}$ to $6 \times 10^{-7}$ torr. The fractional neutralization is given by Eq. (21). The beam is given an initial perturbation by applying a sinusoidal transverse displacement of $10^{-2} \times R_{\mathrm{rms}}\left(5 \times 10^{-3} \mathrm{~cm}\right)$ at the frequency of peak
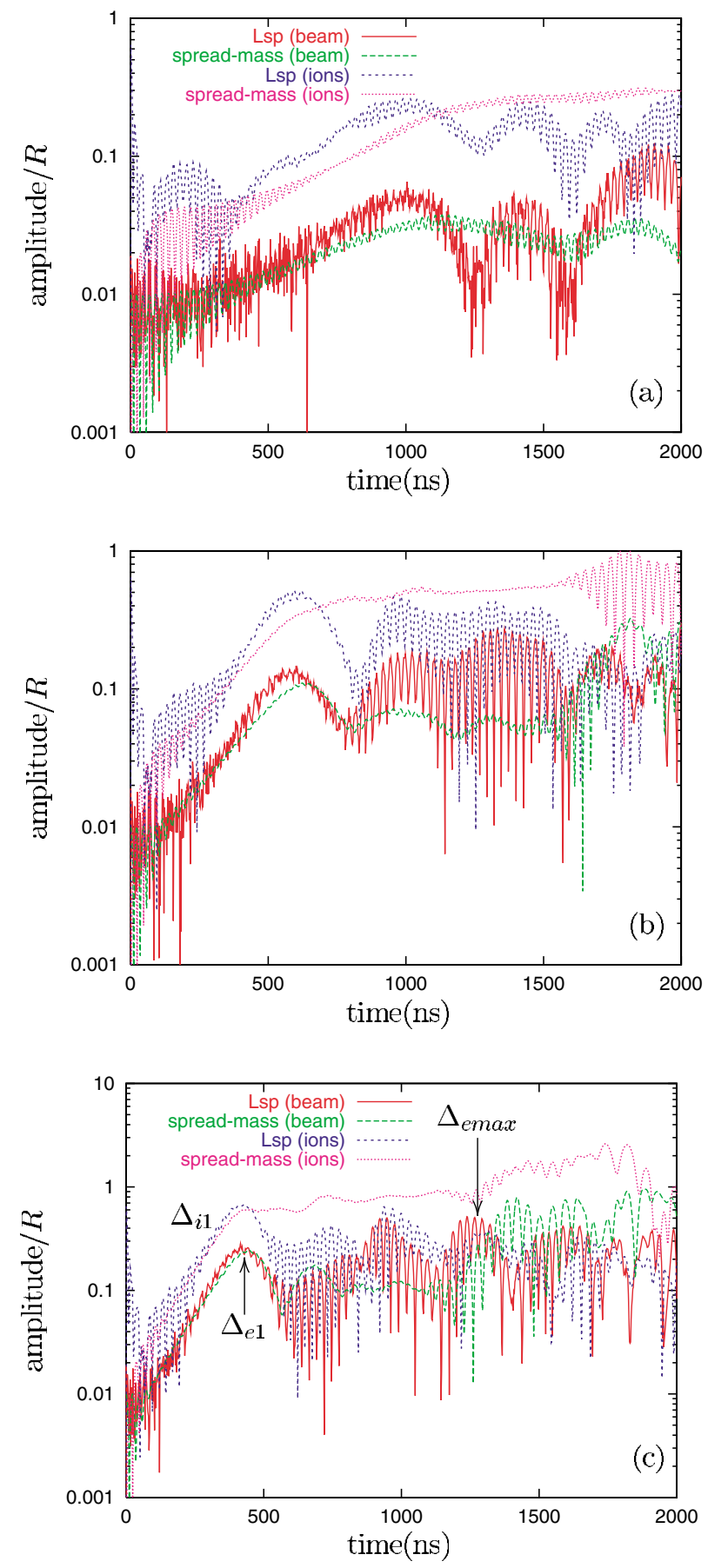

FIG. 5. (Color) Comparison of beam and channel centroids at the end of a $50-\mathrm{m}$ drift, as calculated by the spread-mass and LSP calculations, at pressures of (a) $10^{-7}$ torr, (b) $3 \times 10^{-7}$ torr, and (c) $6 \times 10^{-7}$ torr. The locations of the peaks $\Delta_{e 1}, \Delta_{i 1}$, and $\Delta_{e \max }$ are shown in (c) for the LSP calculation. linear growth $(21.6 \mathrm{MHz})$ as the beam enters the drift space. The transverse beam and channel displacements at the end of the 50-m drift space are plotted in Fig. 5, for three pressures. The agreement between the spread-mass and PIC models is good through the linear and early nonlinear regimes.

The approximate ratio between the ion and beamcentroid amplitudes [Eq. (22)] holds through the linear and early nonlinear behavior. The most important nonlinear effect is the amplitude dependence of the ion oscillation frequency in the transverse electric field of the Gaussian beam density profile. Linear growth proceeds until this effect stops the resonant transfer of energy from the beam to the ions. Both spread-mass and PIC models show some subsequent growth, at a much-reduced rate, indicating that the system is still unstable. In Fig. 6, we compare three values of the centroid amplitude at the end of the drift space from the two models: the beam and ion centroids at the first nonlinear peak $\left(\Delta_{e 1}\right.$ and $\Delta_{i 1}$ in
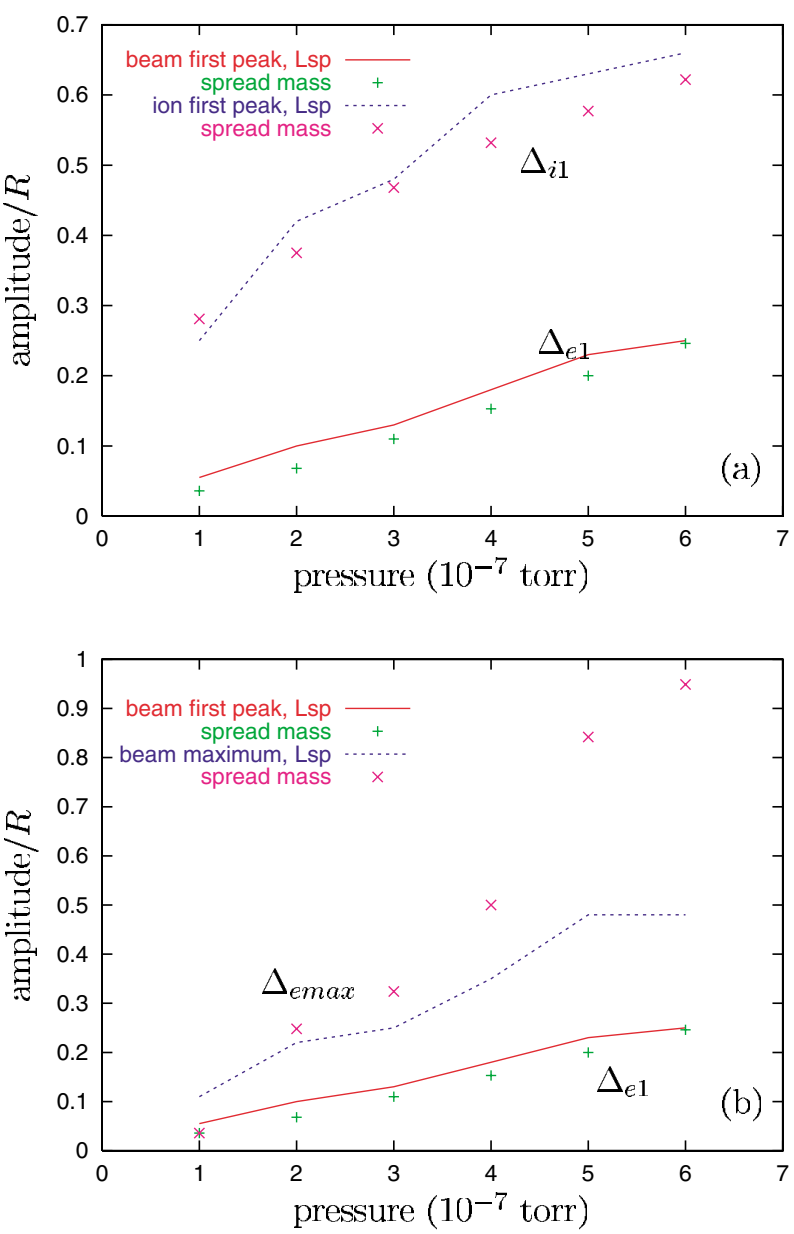

FIG. 6. (Color) Comparison of centroid displacements at the end of the drift space from LSP and spread-mass models. (a) compares the first nonlinear peak for the beam $\left(\Delta_{e 1}\right)$ and ions $\left(\Delta_{i 1}\right)$. (b) shows the first $\left(\Delta_{e 1}\right)$ and maximum $\left(\Delta_{e \max }\right)$ peaks for the beam. 
Fig. 5), and the beam centroid at the maximum offset, whenever in the pulse it occurs $\left(\Delta_{e \max }\right.$ in Fig. 5). We see that there is an approximately linear dependence of $\Delta_{e 1}$ on the pressure.

The good agreement between the spread-mass and PIC models, which have very different treatments of the beam dynamics, is evidence that the main linear and early nonlinear features of the system are being calculated correctly within the set of assumptions common to both models. The codes begin to diverge as the nonlinear stage develops, with the spread-mass treatment showing more growth than the PIC calculation. This may be due to the nonlinear changes in the beam and channel distribution functions (cf. Fig. 11 below). The mass distribution in the spread-mass model [Eqs. (7) and (8)] is chosen to reproduce the linear response of the initial beam and ion configuration. It depends on the shape of the equilibrium charge distribution [3]. It may be possible to improve the agreement in Fig. 6(b) by recalculating the mass distribution as the ions and electrons evolve nonlinearly.

\section{PIC SIMULATIONS FOR ACCELERATED BEAM}

In the DARHT-2 accelerator, the beam is accelerated from 3.2 to $20 \mathrm{MV}$ over 88 gaps [1] (Fig. 1). Over the first eight "injector" cells, the beam is compressed from a large radius $(\sim 9 \mathrm{~cm})$ to a radius of about $1 \mathrm{~cm}$ at the start of the accelerator. We neglect this region in the present calculations as there are several possible transport tunes through this part of the machine [11]. The following simulations start at the entrance to the first accelerating cell block, when the energy is $4.6 \mathrm{MV}(\gamma \simeq 10)$.

The magnetic field increases down the accelerator in a trade-off between beam breakup and "corkscrew" growth [12]. As a result, the equilibrium beam radius decreases according to

$$
R^{2}=\frac{\frac{8 \nu}{\gamma}+\sqrt{\left(\frac{8 \nu}{\gamma}\right)^{2}+16 \hat{B}^{2} \varepsilon^{2}}}{4 \hat{B}^{2}} \simeq \frac{\varepsilon}{\hat{B}}, \nu
$$

where $\hat{B} \equiv e B / m c^{2}$.

Because of the dips in the magnetic field at accelerating gaps, and between adjacent cell blocks, the axial profile has considerable spatial variation, as shown in Fig. 7.

The axial grid size used in the simulations is too large to resolve this structure, so we use a smooth average field, also shown in Fig. 7. The betatron wavelength of the beam varies from 120 to $240 \mathrm{~cm}$, and is always larger than the spatial period of the magnetic field $(\approx 50 \mathrm{~cm})$. In the same spirit, the discrete accelerating gaps are replaced by a spatially uniform axial electric field.

We use a multispecies model of the residual background gas, based on residual-gas analyzer (RGA) data from an accelerating-gap test stand at LANL [8]. The dominant neutral species has an atomic mass unit (AMU) corresponding to $\mathrm{H}_{2} \mathrm{O}$. Lower RGA peaks appear

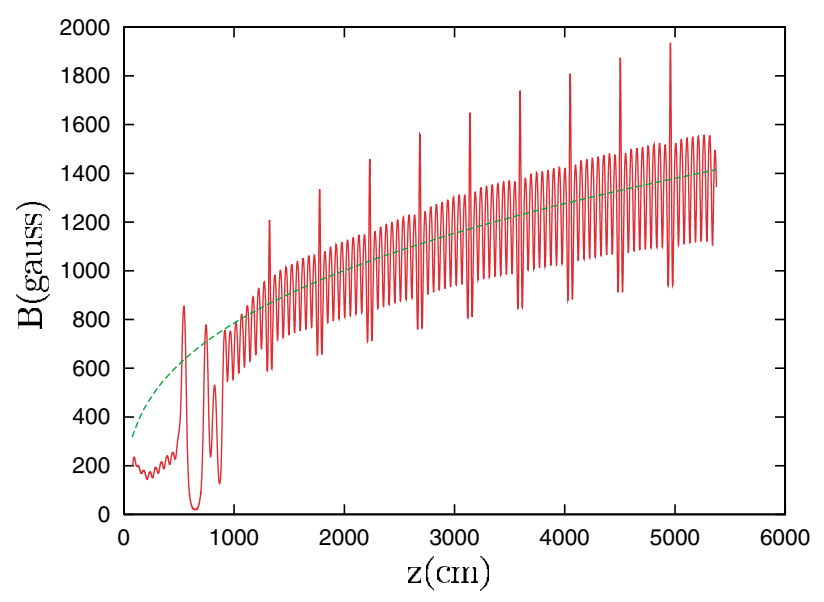

FIG. 7. (Color) Comparison of actual and smoothed axial magnetic field profile. Simulations start at $z \approx 1000 \mathrm{~cm}$.

to correspond to species produced by ionization-induced dissociation of $\mathrm{H}_{2} \mathrm{O}$, namely, $\mathrm{HO}^{+}, \mathrm{O}^{+}$, and $\mathrm{H}^{+}$. We base this on the observation that the relative magnitudes of the RGA data at the AMU values for these species (assuming singly ionized states) correspond roughly to the ionization cross-section ratios derived from Ref. [13]. In addition to $\mathrm{H}_{2} \mathrm{O}$, there is a peak at an AMU value of 28 , which could be either $\mathrm{N}_{2}$ or $\mathrm{CO}$. This peak is about a factor of 30 smaller than that for $\mathrm{H}_{2} \mathrm{O}$. There are several lower signals in the RGA data, which we ignore.

Rather than having a model for dissociation of $\mathrm{H}_{2} \mathrm{O}$ due to electron impact, we initialize the simulations with neutral $\mathrm{H}_{2} \mathrm{O}, \mathrm{HO}, \mathrm{O}, \mathrm{H}$, and $\mathrm{N}_{2}$ in proportion to the partial pressures inferred from the RGA data, and provide single-ionization cross sections for each of these species. In this way, the correct ratios of the ion species are produced. The high-energy cross sections used are given in Table II.

To obtain these, we extrapolated the cross-section data in Ref. [13] from the 1-kV region to the beam energy ( $\geq 4.6 \mathrm{MV}$ ) by fitting to the Bethe cross-section formula (see, e.g., Ref. [14]).

As in the benchmark calculations in Sec. II, we carry out a series of six 3D simulations for pressures ranging from $10^{-7}$ to $6 \times 10^{-7}$ torr. The accelerated beam parameters are given in Table III. For a beam slice near the head of the beam where there is little effect from the

TABLE II. High-energy cross sections for electron impact ionization

\begin{tabular}{cc}
\hline \hline Species & $\sigma_{i}\left(\mathrm{~cm}^{2}\right) \times 10^{-18}$ \\
\hline $\mathrm{H}_{2} \mathrm{O}$ & 0.95 \\
$\mathrm{HO}$ & 0.28 \\
$\mathrm{O}$ & 0.051 \\
$\mathrm{H}$ & 0.12 \\
$\mathrm{~N}_{2}$ & 1.05 \\
\hline \hline
\end{tabular}


TABLE III. Parameters for accelerated beam simulations.

\begin{tabular}{lc}
\hline \hline \multicolumn{1}{c}{ Parameters } & Values \\
\hline Beam relativistic factor $(\gamma)$ & $10-40$ \\
Beam current & $2 \mathrm{kA}$ \\
Beam pulse length & $2 \mu \mathrm{s}$ \\
Beam rms radius & $0.65-0.4 \mathrm{~cm}$ \\
Initial beam emittance & $0.1 \mathrm{~cm} \mathrm{rad}$ \\
Axial magnetic field & $780-1400 \mathrm{G}$ \\
Transport distance & $50 \mathrm{~m}$ \\
\hline \hline
\end{tabular}

weak ion channel, variations of the rms radius and normalized emittance down the accelerator are shown in Fig. 8.

Both behave as expected. The radius varies according to Eq. (32), and the normalized emittance is constant.

Instead of the single-frequency perturbation used in the benchmark calculations to perturb the beam as it enters the accelerator, we use a random, flat broadband spectrum of excitation between $0-100 \mathrm{MHz}$. This is a more realistic perturbation and allows us to see the nonlinear effect of mode competition. The transverse beam
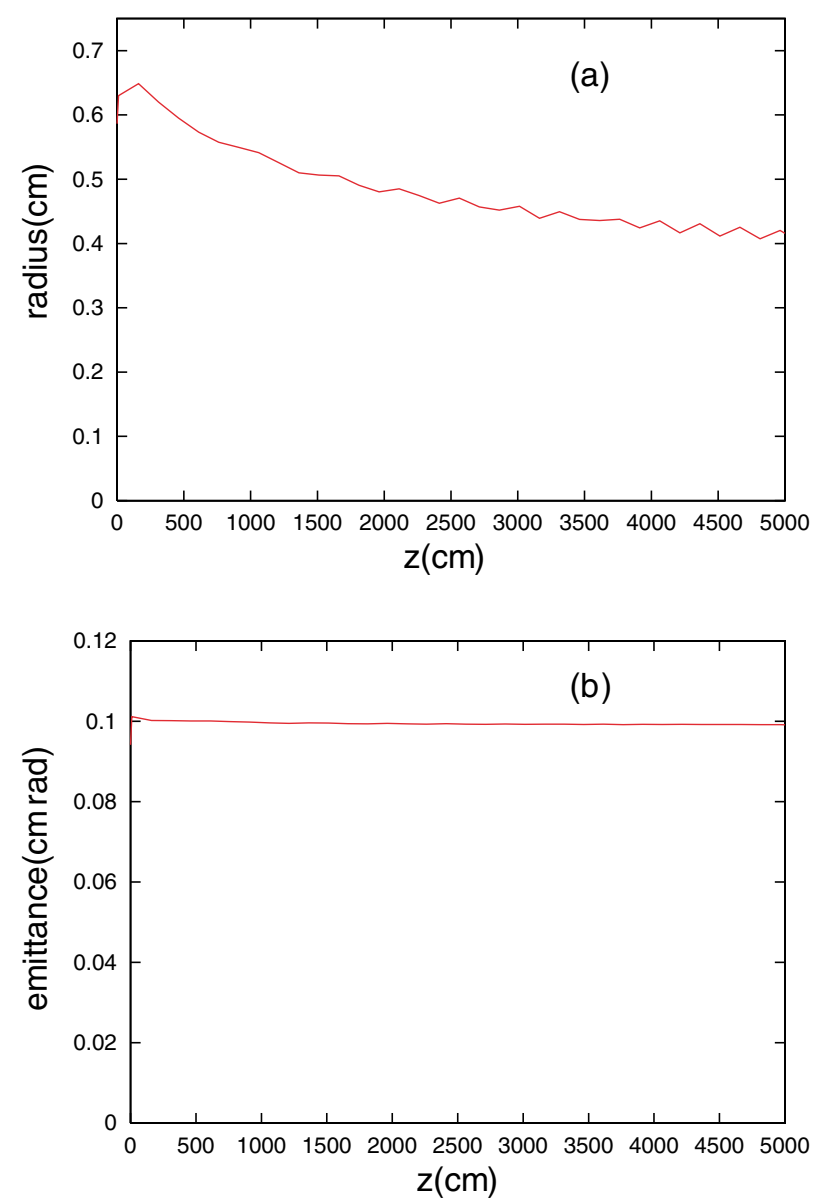

FIG. 8. (Color) Beam radius and emittance as functions of axial position when ion background is negligible. displacement at the end of the $50-\mathrm{m}$ accelerator is shown in Fig. 9 for $10^{-7}, 3 \times 10^{-7}$, and $6 \times 10^{-7}$ torr. As in the case of the drifting beam, the beam displacement is several times smaller than the ion displacement at initial nonlinear saturation.

In Fig. 10, we plot the first nonlinear peak for the beam $\left(\Delta_{e 1}\right)$, channel $\left(\Delta_{i 1}\right)$, and the maximum peak for the beam $\left(\Delta_{e \max }\right)$ as functions of pressure. The ratio $\Delta_{i 1} / \Delta_{e 1}$
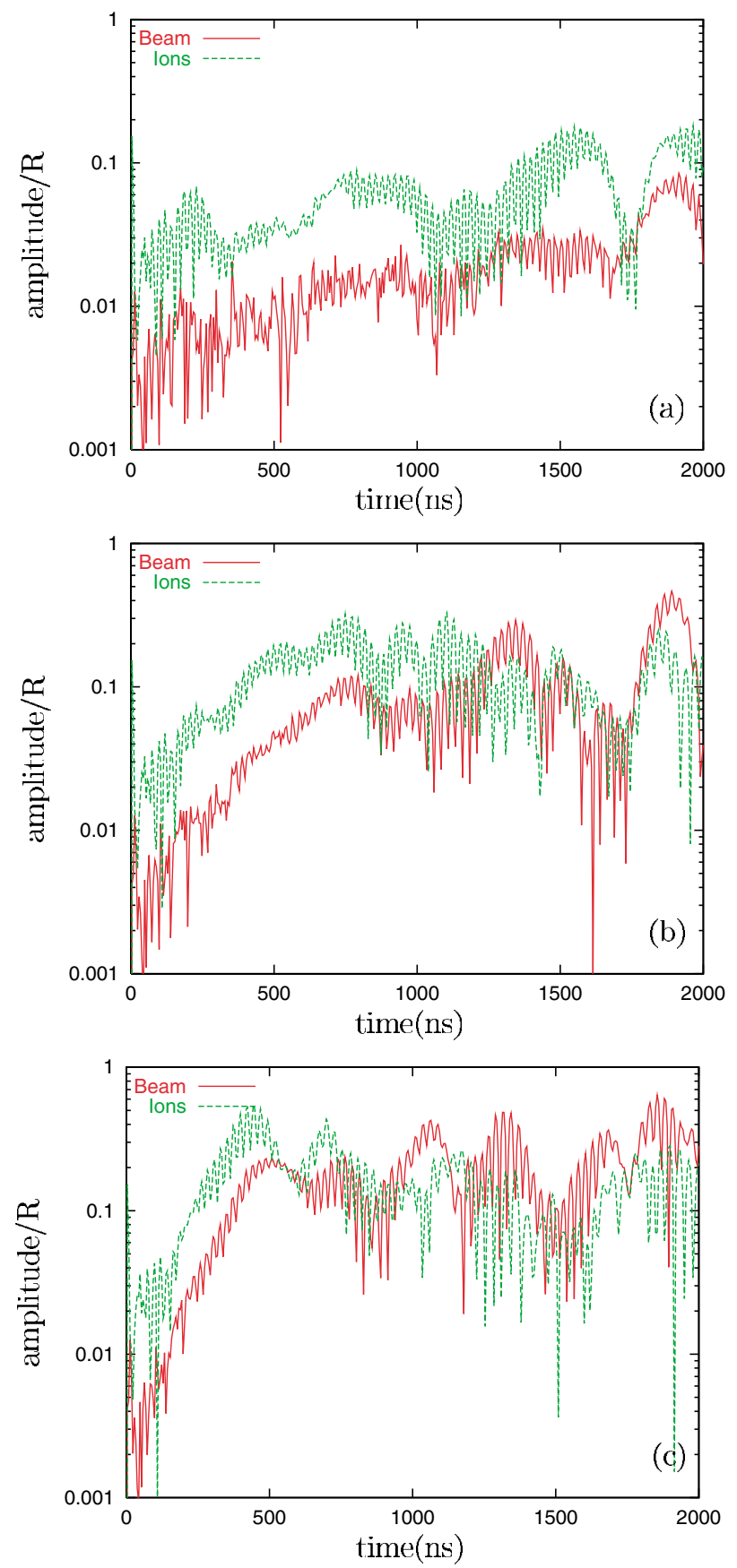

FIG. 9. (Color) Comparison of beam and channel centroids at the end of the $50-\mathrm{m}$ accelerator from LSP calculations, at pressures of (a) $10^{-7}$ torr, (b) $3 \times 10^{-7}$ torr, and (c) $6 \times$ $10^{-7}$ torr. 

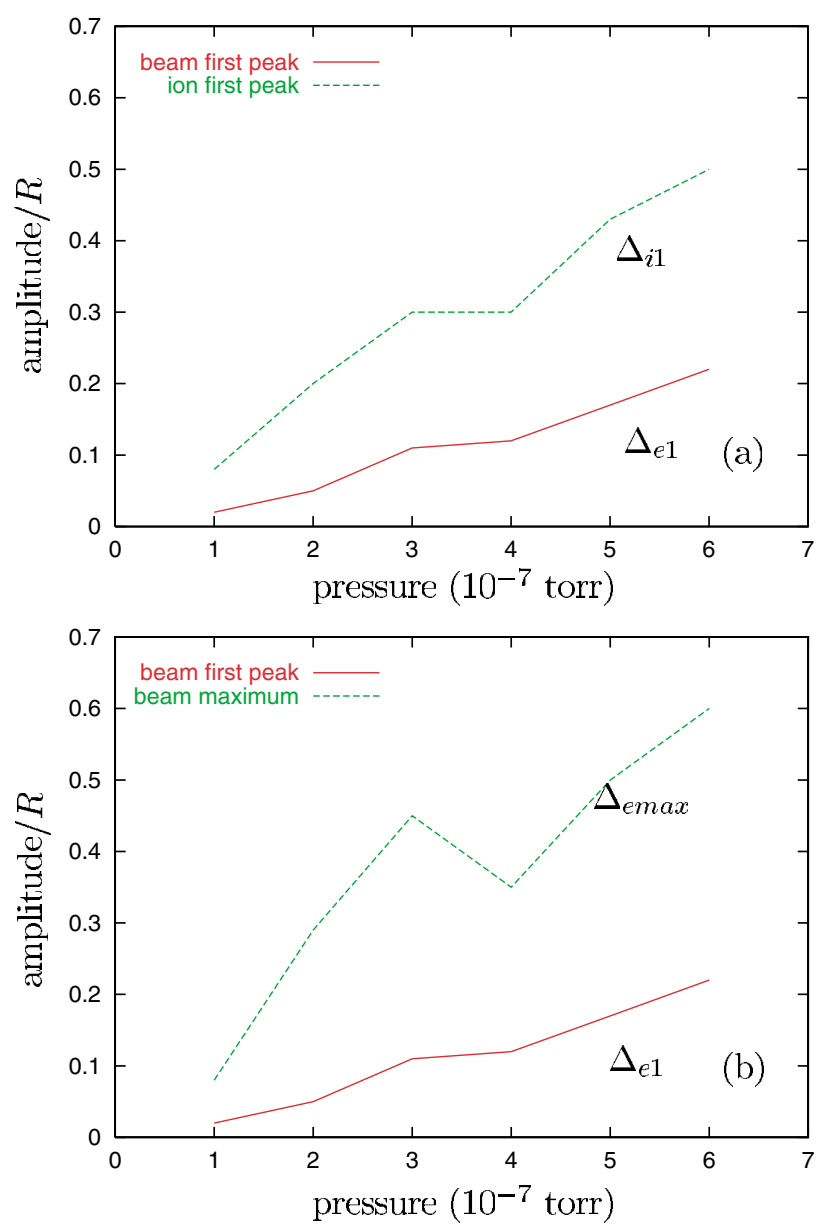

FIG. 10. (Color) Peak beam and ion channel centroid displacements at the end of an accelerator from LSP. (a) shows the first nonlinear peak for the beam $\left(\Delta_{e 1}\right)$ and ions $\left(\Delta_{i 1}\right)$. (b) shows the first $\left(\Delta_{e 1}\right)$ and maximum $\left(\Delta_{e \max }\right)$ peaks for the beam.

shows a similar trend versus pressure as the benchmark runs in Fig. 6. As in the benchmark runs, there is a large decrease in the growth rate after the first nonlinear peak. Subsequent nonlinear peaks are typically 3-4 times larger than the first peak. Even at the highest pressure we have simulated $\left(6 \times 10^{-7}\right.$ torr $)$, however, the maximum beam displacement is less than the beam rms radius.

The effect of the ion-hose instability on the beam radius and emittance is shown in Fig. 11. The instantaneous and 50-ns averages are indicated for both quantities. Of particular interest is the 50-ns average because pulses of this length will be extracted from the $2-\mu$ s pulse to make radiographs [1]. The normalized transverse emittance is defined as [15]

$$
\varepsilon=\frac{1}{4}\left(\operatorname{det} \sum\right)^{1 / 4}
$$

where $\sum$ is the $4 \times 4$ covariance matrix with elements $\left\langle u_{i} u_{j}\right\rangle$, where the vector $\vec{u}=\left(x, y, p_{x}, p_{y}\right)$ is the transverse phase coordinate of a particle, and \langle\rangle denotes an average over a beam slice. For an axisymmetric beam distribu-
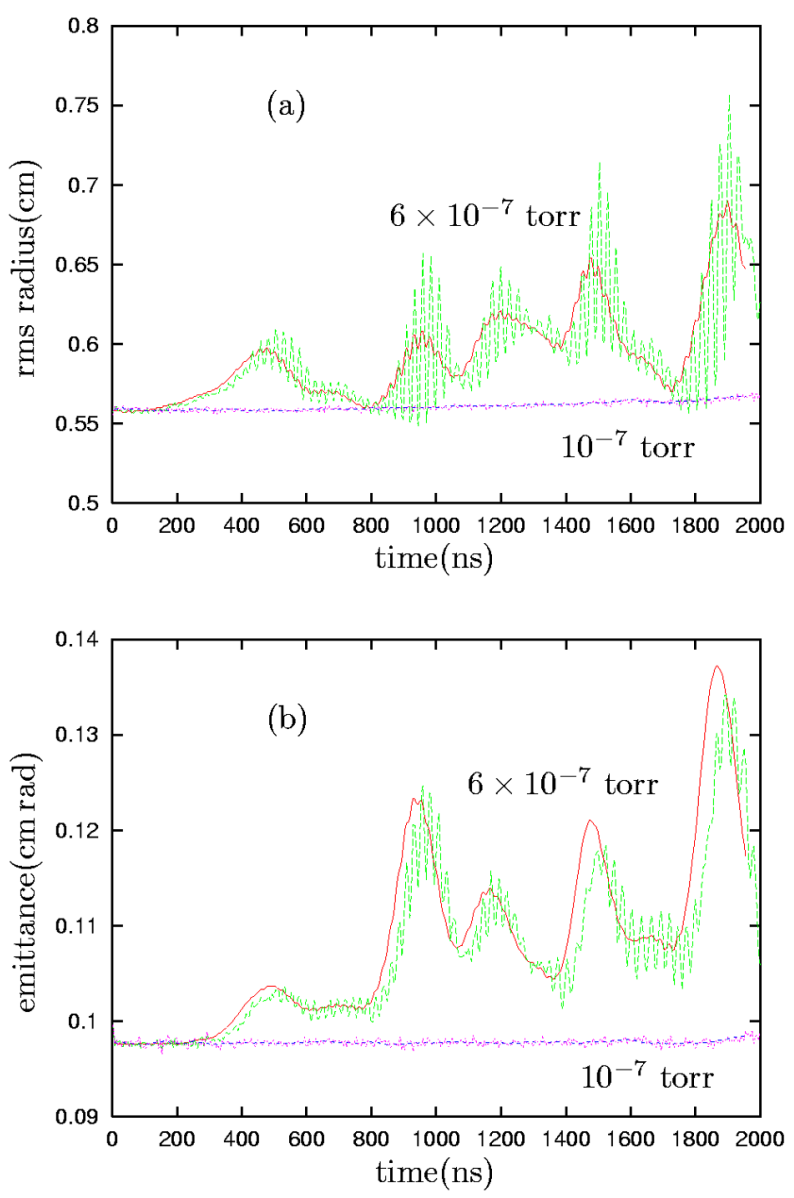

FIG. 11. (Color) Effect of ion-hose instability on beam radius and emittance at the end of the accelerator from the LSP simulations. (a) shows rms radius and (b) shows normalized emittance for pressures of $10^{-7}$ and $6 \times 10^{-7}$ torr. The red lines show the values averaged over $50 \mathrm{~ns}$.

tion, this reduces to the usual definition [16], while, for a beam with no $x-y$ coupling, it reduces to the square root of the product of the $x$ and $y$ emittances [15].

At $10^{-7}$ torr, there is little effect on either the rms radius or the normalized emittance. At $6 \times 10^{-7}$ torr, there is roughly a $20 \%$ increase in the rms beam radius and a $40 \%$ increase in the beam emittance for beam slices which experience the largest ion-hose growth through the accelerator.

\section{CONCLUSIONS}

We have carried out a study of the ion-hose instability for a long-pulse electron beam where the ions are generated by beam-impact ionization of the background gas. For the cases of interest, the instability is in the "strongfocusing" regime where the force, due to the ion channel, is small compared to that provided by the focusing solenoids. Because the ionization fraction $f$ is approximately constant in the vicinity of a given beam slice, the linear amplification can be computed approximately by 
substituting the value of $f$ at the beam slice into the analytic, constant- $f$ expression. The linear theory predicts that the ion channel amplitude is of the order of 4-5 times that of the electron beam.

Nonlinear calculations indicate a large decrease in growth rate when the relative displacement between the beam and ion centroids is sufficiently large. The stabilization is due to the amplitude dependence of the ion oscillation frequency in the nonlinear electric field of the beam. Because the ion oscillation amplitude is several times larger than that of the beam, stabilization can occur when the beam amplitude is a small fraction of the beam radius.

Applied to the DARHT-2 accelerator, the calculations predict that a vacuum pressure $<1.5 \times 10^{-7}$ torr is necessary to keep the beam oscillation amplitude less than about $20 \%$ of the beam rms radius throughout the pulse.

\section{ACKNOWLEDGMENTS}

These calculations were motivated by a treatment of linear ion-hose growth in DARHT-2 by R. J. Briggs. We thank Dan Prono (LANL) for encouraging us to pursue this work, which was supported by Los Alamos National Laboratory. The LSP calculations were carried out on the SGI Origin cluster (Nirvana) at LANL. We thank Sue Welch for her help with the preparation of this article.

[1] M. J. Burns, B. E. Carlsten, H. A. Davis, C. A. Ekdahl, C. M. Fortgang, B. T. McCuistian, F. E. Merrill, K. E.
Nielsen, C. A. Wilkinson, K. P. Chow, W. M. Fawley, H. L. Rutkowski, W. L. Waldron, S. S. Yu, G. J. Caporaso, Y.-J. Chen, E. G. Cook, S. Sampayan, J. A. Watson, G. A. Westenskow, and T.P. Hughes, in Proceedings of the 2001 Particle Accelerator Conference, Chicago, IL (IEEE, Piscataway, NJ, 2001).

[2] R. J. Briggs (unpublished).

[3] E. P. Lee, Phys. Fluids 21, 1327 (1978).

[4] H. L. Buchanan, Phys. Fluids 30, 221 (1987).

[5] P. J. Channell, Phys. Rev. ST Accel. Beams 5, 114401 (2002).

[6] R. Cappi, M. Giovannozzi, E. Metral, G. Metral, G. Rumolo, and F. Zimmerman, Phys. Rev. ST Accel. Beams 5, 094401 (2002).

[7] K. J. O’Brien, J. Appl. Phys. 65, 9 (1989).

[8] D. Prono (private communication).

[9] LSP is a software product of Mission Research Corporation (http://www.mrcabq.com).

[10] D. R. Welch and T. P. Hughes, Phys. Fluids B 5, 339-343 (1993).

[11] T. P. Hughes, D. P. Prono, W. M. Tuzel, and J. R. Vananne, in Proceedings of the 2001 Particle Accelerator Conference, Chicago, IL (Ref. [1]).

[12] G. J. Caporaso and A. G. Cole, in Proceedings of the 1990 Linear Accelerator Conference, Albuquerque, New Mexico, p. 281 (Los Alamos Laboratory Report No. LA-12004-C, 1991).

[13] M.V.V.S. Rao, I. Iga, and S. K. Srivastava, J. Geophys. Res. 100, 26421 (1995).

[14] F. F. Rieke and W. Prepejchal, Phys. Rev. A 6, 1504 (1972).

[15] D. Chernin, Part. Accel. 24, 29 (1988).

[16] E. P. Lee and R. K. Cooper, Part. Accel. 7, 83 (1976). 